\title{
Effect of optimal filtering parameters for autoregressive model $A R(p)$ on motor unit action potential signal
}

\author{
Ayad Asaad Ibrahim, Mohammed Ehsan Safi, Eyad Ibrahim Abbas \\ Department of Electrical Engineering, University of Technology Iraq, Baghdad, Iraq
}

\begin{tabular}{l} 
Article Info \\
\hline Article history: \\
Received Mar 22, 202 \\
Revised Jul 13, 2021 \\
Accepted Jul 29, 2021 \\
\hline Keywords: \\
Autoregressive \\
Final prediction error \\
Power spectral \\
Robust estimation \\
Simulated EMG
\end{tabular}

Corresponding Author:

Mohammed Ehsan Safi

Department of Electrical Engineering, University of Technology Iraq

Baghdad, Iraq

Email:30165@uotechnology.edu.iq

\begin{abstract}
Error is one element of the autoregressive (AR) model, which is supposed to be white noise. Correspondingly assumption that white noise error is a normal distribution in electromyography (EMG) estimation is one of the common causes for error maximization. This paper presents the effect of a suitable choice of filtering function based on the non-invasive analysis properties of motor unit action potential signal, extracted from a noninvasive method-the high spatial resolution (HSR) electromyography (EMG), recorded during low-level isometric muscle contractions. The final prediction error procedure is used to find the number of parameters in the model. The error signal parameter, the simulated deviation from the actual signals, is suitably filtered to obtain optimally appropriate estimates of the parameters of the automatic regression model. It is filtered to acquire optimally appropriate estimates of the parameters of the automatic regression model. Then appropriate estimates of spectral power shapes are obtained with a high degree of efficiency compared with the robust method under investigation. Extensive experiment results for the proposed technique have shown that it provides a robust and reliable calculation of model parameters. Moreover, estimates of power spectral profiles were evaluated efficiently.
\end{abstract}

This is an open access article under the CC BY-SA license.

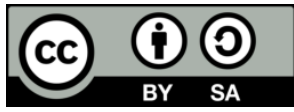

\section{INTRODUCTION}

The surface electromyography (EMG) signal is increasingly used for tension state discrimination in a single muscle or a group of muscles and muscle fatigue measurements [1]. This noise-like signal is an interference pattern that is the temporal and the spatial sum of the action potential (AP) of all motor units (MU) in the region of the detecting electrodes [2]. This signal's statistical properties are related to muscle tension and spectral shape changes with muscle tension changes, but the changes are not significant [3]. The EMG signal is assumed to be a stationary zero-mean gaussian random process [4]. The muscles can change their contraction levels by changing the activated motor units [5]. These changes will cause some outlier samples to appear negatively, affecting the assumed distribution mentioned above [6]. Modeling the motor unit action potential (MUAP) has been particularly useful in developing a mathematical formulation to study, understand, and process techniques for this signal [7]. In this paper, a MUAP signals a model extracted from the IB2 spatial filter [8], based on the autoregressive model coefficients of this signal. Its parameters are derived from real recorded unipolar EMG signals. This model provides the means for generating MUAP signals whose amplitude and spectral characteristics depend on subjects and muscle type under normal contraction to be simulated. Such simulated signals can be used as test signals to evaluate the performance of 
MUAP processing techniques. The degree of sensitivity of the power spectral shapes and suitable choice of robust filtering is investigated when the MUAP signals contain innovation (IO) and additive (AO) outliers, respectively, with processing times 100,400 , and $800 \mathrm{msec}$. The sequential least square method (SLSM) has been used to enhance robust techniques. That is to fit the residuals' optimal distribution to obtain autoregressive time series estimators $(\varnothing)$ for bipolar EMG signal. It is shown that the improvement of the filtering of the asymmetrical type of contamination distribution is (extreme value distribution-smallest values). For all EMG signals after filtering (normal and abnormal signals), the error signal was fit to the Cauchy distribution function. To obtain specific additives for robust methods in the field of installing an optimal residuals distribution through the use of the sequential small square method (SLSM), to obtain an autoregressive time series estimator $(\varnothing)$ depends on using probability plot (PP) inspection method [9]. Standardized residuals evaluate the principle of the (PP) method according to the scale parameter estimator of the order statistics for the suggested distribution and the inverse probability of the cumulative distributions that had been related to the assumed distribution [10]. Asymptotic formula to enable the application.

A variety of new methods are proposed to improve EMG signal estimation in the last few years. Sommer et al. [11], proposed four techniques to estimate the elbow joint angle from surface EMG. These methods are autoregressive with exogenous input, state space, autoregressive moving-average with exogenous input, autoregressive integrated moving-average with exogenous input. After the model was selected, a second experiment was performed in order to validate the estimation procedure. Furui et al. [12], presents an estimation method based on variance distribution and marginal likelihood maximization. The experiment results show that the proposed algorithm is more accurate than the Gaussian-based approach. Wright and Stashuk [13] describe a new algorithm to estimate motor unit potential. The proposed method is based on kernel weighted ensemble averaging supported by dynamic time wrapping (DTW). The experiment results show significant improvement compared with a spike-triggered average. Sommer and Cordero [14], proposed an algorithm to estimate EMG signal based on the Hammerstein-wiener with wavelet network. The results show low root-mean-square errors (RMSE) $\left(10.82 \pm 3.73^{\circ}\right)$ and high correlations $(94.90 \pm 92 \%)$ to the measured data. Kline and Luca [15], present a method for error reduction contain multiple decomposition estimates based on the trade-off between the yield of MUAP trains obtained from a given accuracy level and the time of decomposition. All these researches suppose that the error signal is white noise.

\section{OPTIMAL FILTERING METHODS}

The idea of optimum spectral density estimate according to the suggested distribution makes no difference based on the procedures related to the robust filtering method proved by Thomson [16]. Nevertheless, with the addition of the weight function generated by specific distribution asymptotic with the shape of normal distribution in the average area, the edges are heavier and thicker than normal distributions [17]. Since the MUAP data have outliers, the assumption that the residual of AR(p) has a normal distribution is violated. The ability of the extension of the robust filtering procedure method as a result of multicontamination types, which reflect several kinds of outliers, which are either upper or lower outliers, or within the sampling observation [18]. In consequence, two types of contaminating distribution can be characterized. Table 1 shows weighted filter formulas concerning the standardized cumulative density according to the specific distribution mentioned in [19].

Table 1. Weighted filter formulas concerning the standardized cumulative density

\begin{tabular}{cc}
\hline Adjusted Distribution Functions & Filter Weighted Function \\
\hline Adjusted Extreme Value-Smallest & $w(u)=\exp [-\exp (-(q(|u|-q)))]$ \\
Adjusted Extreme Value-Largest & $w(u)=\exp [-\exp ((q(|u|-q)))]$ \\
Adjusted Cauchy & $w(u)=0.5+\pi^{-1} \tan ^{-1}(q(|u|-q))$ \\
Adjusted Logistic & $w(u)=1 /[1+\exp [-(q(|u|-q))]]$ \\
Adjusted Double Exponential & $w(u)=\left\{\begin{array}{l}1-0.5 \exp [-(q(|u|-q))]: \text { if } \geq a_{\text {if }} \geq \mathrm{a} \\
0.5 \exp [q(|u|-q)]: o . w .\end{array}\right.$ \\
Adjusted Normal & $\left.w(u)=0.5\left[1+\left\{1-\exp (-2(q(|u|-q)))^{2} / \pi\right)\right\}^{0.5}\right]$ \\
\hline
\end{tabular}

Where:

$w(u)$ : is the weighted filter

$u$ : is the residual random variable

$q$ : is a parameter and $=\varphi_{N(0,1)}^{-1}(1-1 / N)$

$\varphi_{N(0,1)}:$ the natural cumulative density function

$N$ : number of samples per interval 


\subsection{Symmetric contaminate distribution}

The $G(*)$ reflects any symmetric distribution, syncs about the centroid of the observed symmetric distribution $(F: N(\mu, \sigma 2))$, and subsequently $G(*)$ becomes $(G:(\mu, a \sigma 2))$. In the event of a state shift $G(*)$ would be $(G:(\mu \pm b, a \sigma 2))$. The symmetric alternative to the normal distribution as a logistic, double exponential, Cauchy, and normal distribution has been chosen.

\subsection{Asymmetric contaminate distribution}

The $G(*)$ reflects any asymmetric distribution about every other point distinctive from the location of $F(*)$ distribution. The center point of $G(*)$ will differ from that of $(\mu)$ and will also differ from that of $F(*)$. Accordingly, we have chosen the alternative asymmetric distributions.

A preliminary test for the fit of the residual distributions was performed to estimate the formula for the optimum weight. The standardized commutative density of the residual distribution is thought to be more advantageous than the other standard cumulative densities expected for residual sets, which is referred to as robust filtering. As we mentioned above, Table 1 shows weighted filter formulas concerning the standardized cumulative density according to the specific distribution mentioned in [19].

The different methods are compatible with the distribution of residual grade and the degree of synthesis or determination of the significance of critical points for appropriate test statistics and give the rigorous process of examination, comparison, and evaluation among the various proposed distributions. As suggested in [17], the probability plot approach and appropriate parameters (for example, single correlation coefficient measurement and goodness of fit according to static (SR)) were chosen.

$$
\begin{aligned}
& r_{\left.R_{(t)} \cdot K_{t}\right)}=\frac{\sum_{t=p-1}^{n}\left(K_{t}-K\right) \cdot\left(R_{(t)}-R\right)}{\left(\sum_{t=p-1}^{n}\left(K_{t}-K\right)^{2} \cdot \sum_{t=p-1}^{n}\left(R_{(t)}-R\right)^{2}\right)^{0.5}} \\
& r_{\left.\left.R_{(t)} \cdot K_{t}\right)\right)}=\frac{\sum_{t=p-1}^{n}\left(K_{t}-K\right) \cdot\left(R_{(t)}-R\right)}{\left(\sum_{t=p-1}^{n}\left(K_{t}-K\right)^{2} \cdot \sum_{t=p-1}^{n}\left(R_{(t)}-R\right)^{2}\right)^{0.5}}
\end{aligned}
$$

$\mathrm{R}(\mathrm{t})$ : is the order statistics of standardized residual according to the assumed distribution scale parameter. $\mathrm{K}$ : denotes the inverse probabilities of the cumulate sample distribution with respect to the assumed distribution. Figure 1 shows the flowchart of the robust optimal filter algorithm for the suggested method.

\subsubsection{The studied distributions}

Table 2 shows the arithmetical formula for the probability plot coordinate method, representing the inverse probabilities of the cumulative sampling of non-normal distributions.

$$
F^{-1}\left(\frac{t}{n+1}\right) \quad \text { or } \quad F^{-1}\left(\frac{t-0.5}{n}\right)
$$

And the standardized residuals' order statistics:

$$
R_{(r)}=\left({ }^{\varepsilon_{(t)}} /(S . P)\right)
$$

where $\mathrm{SP}=$ scale parameter [19], [20]. The standardized cumulative density function cannot be immediately evaluated to yield $\mathrm{F}(\mathrm{u})$ with respect to the random variable $(\mathrm{w})$.

$$
F(u)=(2 \pi)^{-0.5} \int_{-a}^{u} \exp \left(-0.5 w^{2}\right) d w
$$

Table 2. The inverse probabilities of the cumulative sampling sistribution functions

\begin{tabular}{cc}
\hline Adjusted Distribution Functions & Filter Weighted Function \\
\hline Adjusted Extreme Value-Smallest & $w(u)=\exp [-\exp (-(q(|u|-q)))]$ \\
Adjusted Extreme Value-Largest & $w(u)=\exp [-\exp ((q(|u|-q)))]$ \\
Adjusted Cauchy & $w(u)=0.5+\pi^{-1} \tan ^{-1}(q(|u|-q))$ \\
Adjusted Logistic & $w(u)=1 /[1+\exp [-(q(|u|-q))]]$ \\
Adjusted Double Exponential & $w(u)=\left\{\begin{array}{l}1-0.5 \exp [-(q(|u|-q)))]: \text { if } \geq a \\
0.5 \exp [q(|u|-q)]: o . w . \text { if u } \geq \mathrm{a}\end{array}\right.$ \\
Adjusted Normal & $\left.w(u)=0.5\left[1+\left\{1-\exp (-2(q(|u|-q)))^{2} / \pi\right)\right\}^{0.5}\right]$ \\
\hline
\end{tabular}




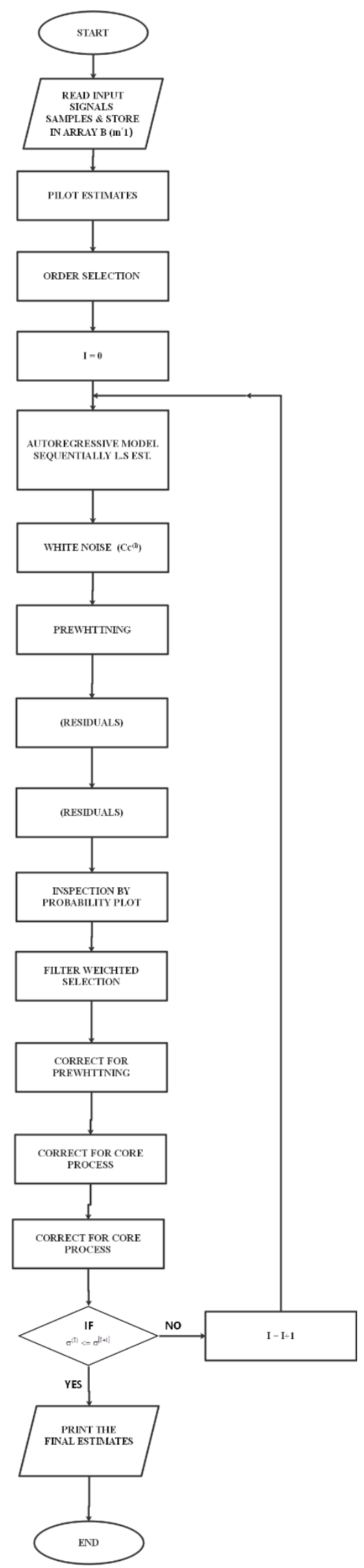

Figure 1. The flowchart of the proposed methodology algorithm 
One of the following approximate equation was chosen to solve (5) [11]:

$$
F(u) \approx 0.5\left(1+\left(1-\exp \left(-2 u^{2} / 2 \pi\right)\right)^{2}\right)
$$

at which $\mathrm{u}=2.807,3.090$, and 3.480 for 100,250 , and $400 \mathrm{msec}$ windows respectively at maximum error $\approx 0.003$. According to the above, a new equation was derived by [9]:

$$
\begin{aligned}
& \text { Let } F(u)=Y \\
& u=\left(\pi / 2\left(-\ln \left(1-(2 y-1)^{2}\right)\right)\right)^{0.5} \\
& \text { Iven } F^{-1}(u)=\left((\pi / 2) \cdot\left(-\ln \left(1-(2 u-1)^{2}\right)\right)\right)^{0.5} \\
& F^{-1}\left(\frac{t}{n+1}\right)=\left((\pi / 2)\left(-\ln \left(1-\left(\frac{2 t-n-1}{n+1}\right)^{2}\right)\right)\right)^{0.5}
\end{aligned}
$$

The inverse probability of normal cumulate sampling distributions functions:

$$
F^{-1}\left(\frac{t-0.5}{n}\right)=\left((\pi / 2)\left(-\ln \left(1-\left(\frac{2 t-n-1}{n}\right)^{2}\right)\right)\right)^{0.5}
$$

for each value of $(T)$, we obtain $\pm K_{t}$ at the failed $u=0$.

The flowchart of the proposed method algorithm is shown in Figure 1. It started with the data acquisition of the input signals, then the pilot estimation to select the order of the AIC criterion. A.R by sequentially LS estimation to calculate the error to set weight while the probability plot is inspected. The subject's subroutine performs the calculation of the distribution function of weight. Consequently, the correction of pre-whitening is performed. Finally, the minimum white noise value is selected.

\section{THE PROPOSED METHOD}

Figure 2 shows a block diagram of the proposed method for system configuration. Four subjects, males and females, different types of muscles, flexor, extensor, and Abductor Pollicis Brevis, are used in this investigation. These correspond to the tensions generated by the subjects. A two-dimensional arrangement of the measuring electrode, high spatial resolution electromyography, is used, designed, and developed by the Institute of Applied Medical Engineering, Helmholtz Institute of RWTH Aachen, and University Hospital Aachen are used. Figure 3 shows the electrode array arrangement.

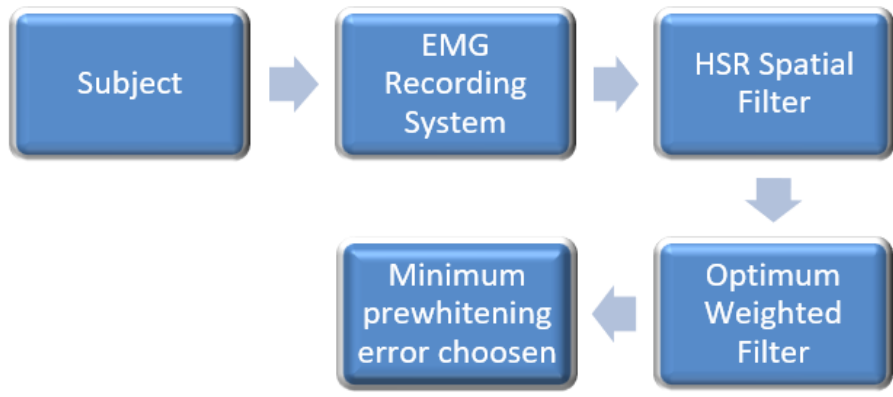

Figure 2. The proposed method of the system setup

These electrodes have a dry surface, connected as a unipolar, with a separation of $2.5 \mathrm{~mm}$. The detected signals are amplified and fed into a laptop via a 16-channels A/D convertor. For each subject and muscle, 5 seconds unipolar 16-channels EMG signals are sampled at a rate of $4 \mathrm{kHz}$ and stored on the laptop. The sampling rate used is quite sufficient, considering the EMG signal bandwidth of about 6-500 Hz [21]. Considering the inter-electrode distance assumed to be $2.5 \mathrm{~mm}$, it determines that the spatial frequencies will be lower than $0.2 \mathrm{~mm}-1$. Since the conduction velocity is $4 \mathrm{~m} / \mathrm{s}$, time frequencies will be lower than $800 \mathrm{~Hz}$ [22]. 


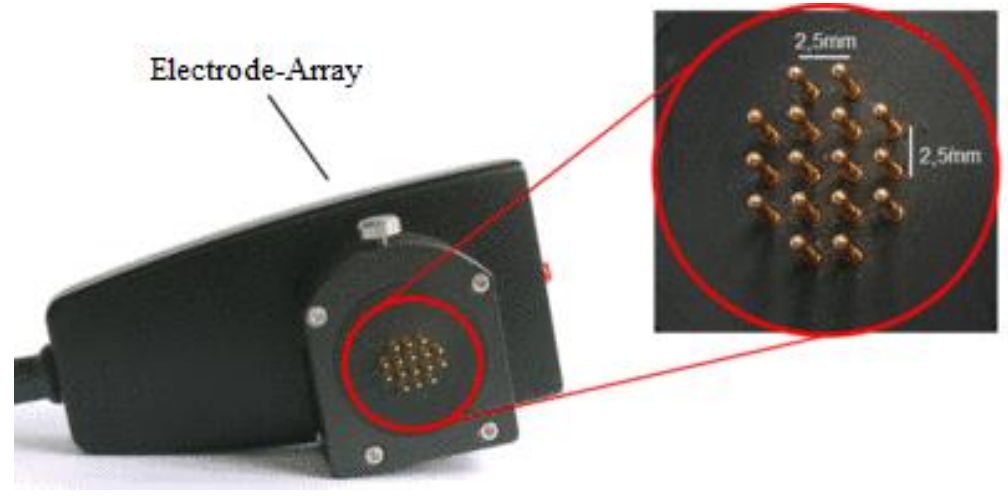

Figure 3. The electrode array arrangement

\subsection{Spatial filter}

For specific and accurate electrophysiological analysis of a single motor unit, it is essential to reduce the area of detection. To overcome this inherent limitation of surface detection, the spatial filter in the form of a one- or two-dimensional grid electrode array is implemented [23] to enhance the recorded signal's spatial selectivity. It has been shown in [8] that the IB2 spatial filter shows that it has a higher spatial resolution in comparison with normal double differentiating (NDD), inverse rectangle (IR), and inverse binomial (IB4). The IB2 spatial filters defined by filter mask in (12). Figure 4 shows the resultant spatially filtered channels recorded simultaneously from a female abductor Pollicis Brevis muscle.

$$
M(x, z)_{I B^{2}}=-\frac{1}{16}\left(\begin{array}{ccc}
1 & 2 & 1 \\
2 & -12 & 2 \\
1 & 2 & 1
\end{array}\right)
$$

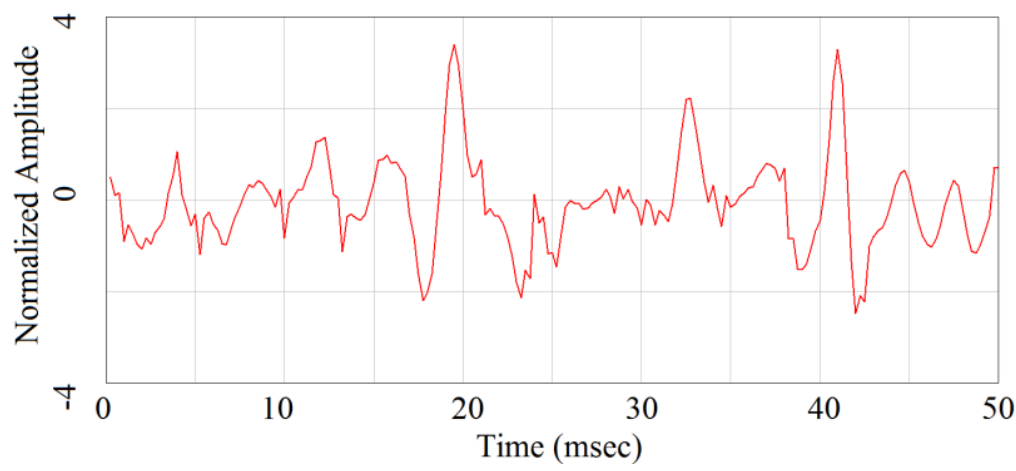

Figure 4. The EMG after filtering with $\mathrm{IB}^{2}$

\subsection{Robust optimality filtering}

These models were considered important due to the various contamination fields, which reflect multiple forms of outliers. These outliers may be distributed to the upper or lower outliers or within the data signal's sampling observation. Consequently, it is essential to differentiate between symmetrical and asymmetrical contamination distributions. Instead of the normal distribution, two symmetrical distributions were chosen, namely the extreme value distribution, which includes the smallest and greatest values.

The normal distribution for the symmetrical distribution was chosen from the cauchy, logistic, and double exponential distributions. Four subjects, different types of muscles, under the normal state of tension with three windows, for each, were chosen in this work. Seven criteria estimated the order of the AR model (P) used for the optimum estimation of the model order. The first was akiake's final predicted error (FPE) criterion. The second technique was akaike's information criterion (AIC). The third was Parzen's criterion autoregressive function (CAT). The fourth was rissanen's minimum description length (RIS). The fifth was the eesidual variance (RV). The sixth was rissanen (MDL). The final one was Hannan and Quinn [24], which 
was used for biomedical applications such as the optimum order of autoregressive models for Hart Rart by Boardman et al. [25] and EEG classification by Atyabi et al. [26]. For the seven techniques, the mean square error and bias change for each model order, the above-defined function is used with a minimum point; the model order $(\mathrm{P})$ corresponding to this minimum point is the optimum for stimulating the MUAP signal for all windows under study. Each set of data was tested using the seven criteria.

The error pre-whitening values $(\varepsilon(z))$ were generated according to the distributed function $G(*)$ to give the superior performance of the simple correlation coefficients (SCC) between the value of the order statistics of the standard error and the value of the standard cumulative data according to the two formulas (3). For each iteration filtering cycle when: $\mathrm{t}=1.2 \ldots ; \mathrm{n}$ : the total number of integers.

The least-square is tested by a probability plot to estimate its distribution to choose the best filtering weight function. This process will be repeated until the last iteration cycle is obtained to decide which probability distribution will be selected for the pre-whitening error at later simulation on the microcomputer. The simulation generates the pre-whitening noise $(\varepsilon(z))$ that will serve as an input to the robust estimator model's function. A normal distribution pre-whitening signal was also generated as an input to the transfer function of the AR (n) parameter, while the output is $(Y(z))$.

\section{EXPERIMENTAL RESULTS}

The optimum estimation of the model's order for the four subjects and time intervals of 100, 400, $800 \mathrm{msec}$ are shown in Table 3. The results obtained show that FPE, AIC, CAT, and R.V. have the optimum estimation of the residual error model order. Irrigation preference is given RV for the stability of the model order's estimation regardless of the duration of the window of investigation. At the same time, RIS shows the worst order estimation for residual error. Table 4 shows the simply correlated coefficients for the four subjects with windows $100,400,800 \mathrm{msec}$ of the optimum distribution for these coefficients. The results show that SCC is stationary for all the studied cases.

Table 3. The optimum estimation of the models' order

\begin{tabular}{|c|c|c|c|c|c|c|c|c|c|c|c|c|}
\hline \multirow{3}{*}{$\begin{array}{c}\text { Model } \\
\text { order }\end{array}$} & \multicolumn{3}{|c|}{ Subject 1} & \multicolumn{3}{|c|}{ Subject 2} & \multicolumn{3}{|c|}{ Subject 3} & \multicolumn{3}{|c|}{ Subject 4} \\
\hline & 100 & 250 & 400 & 100 & 250 & 400 & 100 & 250 & 400 & 100 & 250 & 400 \\
\hline & msec & msec & msec & msec & msec & $\mathrm{msec}$ & $\mathrm{msec}$ & msec & msec & msec & msec & msec \\
\hline FPE & 10 & 10 & 10 & 14 & 14 & 14 & 8 & 7 & 7 & 2 & 3 & 3 \\
\hline AIC & 10 & 10 & 10 & 14 & 14 & 14 & 8 & 7 & 7 & 2 & 3 & 3 \\
\hline CAT & 11 & 11 & 11 & 19 & 20 & 19 & 8 & 8 & 8 & 4 & 4 & 4 \\
\hline RIS & 1 & 1 & 1 & 1 & 1 & 1 & 1 & 1 & 1 & 1 & 1 & 1 \\
\hline RV & 10 & 10 & 10 & 19 & 19 & 19 & 8 & 8 & 8 & 3 & 3 & 3 \\
\hline MDL & 9 & 9 & 9 & 1 & 1 & 1 & 3 & 1 & 1 & 1 & 1 & 1 \\
\hline HQ & 10 & 10 & 10 & 14 & 14 & 14 & 7 & 3 & 3 & 1 & 1 & 1 \\
\hline
\end{tabular}

$\underline{\text { Table 4. SCC estimators of the extreme large values distribution }}$

\begin{tabular}{cccc}
\hline Subjects & Window msec & First Cycle SCC & Last Cycle SCC \\
\hline \multirow{2}{*}{1} & 100 & 0.877 & 0.873 \\
& 250 & 0.865 & 0.875 \\
\multirow{2}{*}{2} & 400 & 0.869 & 0.879 \\
& 100 & 0.812 & 0.824 \\
& 250 & 0.801 & 0.811 \\
3 & 400 & 0.824 & 0.826 \\
& 100 & 0.837 & 0.854 \\
4 & 250 & 0.848 & 0.897 \\
& 400 & 0.851 & 0.873 \\
& 100 & 0.942 & 0.955 \\
& 250 & 0.922 & 0.923 \\
& 400 & 0.901 & 0.927 \\
\hline
\end{tabular}

Table 5 shows the mean square of the pre-whitening errors for all cases at the last cycle of iteration for robust and conventional models. The results show that good improvement has been achieved in all cases. Consequently, the conventional model is still sensitive to the (AO). The results show that the robust method is more efficient than the conventional method. Also, the MUAP of all cases shows that the extreme largest value distribution is the best model for residual error. Figure 5 illustrates the power spectrum for the real and simulated EMG at the last iteration using the extreme most extensive value distribution. Within typical type on generating pre-whitening, noise for the selected subject at $250 \mathrm{msec}$ windows. 
Table 5. The MSE of the pre-whitening MUAP for the conventional and robust method

\begin{tabular}{|c|c|c|c|c|c|c|c|c|}
\hline \multirow{3}{*}{ Subjects } & \multirow{3}{*}{$\begin{array}{l}\text { Window } \\
\text { (msec) }\end{array}$} & \multirow[b]{2}{*}{$\begin{array}{c}\text { Conventional } \\
\text { Method }\end{array}$} & \multicolumn{6}{|c|}{ Robust Method } \\
\hline & & & $\begin{array}{c}\text { Extreme } \\
\text { Smallest } \\
\text { Value }\end{array}$ & $\begin{array}{c}\text { Extreme } \\
\text { Larges } \\
\text { Value }\end{array}$ & Cauchy & Logistic & $\begin{array}{c}\text { Double } \\
\text { Exponential }\end{array}$ & $\begin{array}{c}\text { Normal } \\
\text { Distribution }\end{array}$ \\
\hline & & MSE & MSE & MSE & MSE & MSE & MSE & MSE \\
\hline \multirow[t]{3}{*}{1} & 100 & 0.2658 & $4.991 \mathrm{e}-29$ & 0 & $4.588 \mathrm{e}-32$ & $7.346 \mathrm{e}-38$ & 0.26587 & $1.104 \mathrm{e}-29$ \\
\hline & 250 & 0.2781 & $9.142 \mathrm{e}-29$ & $\mathbf{0}$ & 7.425e-32 & $1.005 e-36$ & 0.27818 & $3.429 \mathrm{e}-28$ \\
\hline & 400 & 0.2624 & $7.064 \mathrm{e}-30$ & $\mathbf{0}$ & $4.030 \mathrm{e}-31$ & $1.206 \mathrm{e}-39$ & 0.26247 & 0 \\
\hline \multirow[t]{3}{*}{2} & 100 & 0.1027 & $3.856 \mathrm{e}-29$ & $\mathbf{0}$ & $4.244 \mathrm{e}-32$ & $7.346 \mathrm{e}-38$ & 0.10276 & $1.109 \mathrm{e}-29$ \\
\hline & 250 & 0.0894 & $9.118 \mathrm{e}-29$ & $\mathbf{0}$ & 7.617e-32 & $1.005 e-36$ & 0.08942 & $3.315 \mathrm{e}-28$ \\
\hline & 400 & 0.1074 & $7.099 \mathrm{e}-30$ & $\mathbf{0}$ & $3.826 \mathrm{e}-31$ & $1.389 \mathrm{e}-39$ & 0.10749 & 0 \\
\hline \multirow[t]{3}{*}{3} & 100 & 0.1733 & $3.978 \mathrm{e}-29$ & $\mathbf{0}$ & $4.129 \mathrm{e}-32$ & $7.346 \mathrm{e}-38$ & 0.17337 & $1.109 \mathrm{e}-29$ \\
\hline & 250 & 0.2378 & $9.142 \mathrm{e}-29$ & $\mathbf{0}$ & $6.869 \mathrm{e}-32$ & $1.005 e-36$ & 0.23794 & $3.437 \mathrm{e}-28$ \\
\hline & 400 & 0.2429 & $7.045 \mathrm{e}-30$ & $\mathbf{0}$ & $4.116 \mathrm{e}-31$ & $1.091 \mathrm{e}-39$ & 0.2493 & 0 \\
\hline \multirow[t]{3}{*}{4} & 100 & 1.1930 & $7.287 \mathrm{e}-29$ & $\mathbf{0}$ & $4.585 \mathrm{e}-32$ & $7.346 \mathrm{e}-38$ & 1.193 & $1.109 \mathrm{e}-29$ \\
\hline & 250 & 1.0834 & $9.538 \mathrm{e}-29$ & 0 & $1.983 \mathrm{e}-31$ & $1.005 e-36$ & 1.0834 & $3.478 \mathrm{e}-28$ \\
\hline & 400 & 0.9573 & $7.099 \mathrm{e}-30$ & $\mathbf{0}$ & $4.209 \mathrm{e}-31$ & $1.036 \mathrm{e}-39$ & 0.95735 & 0 \\
\hline
\end{tabular}

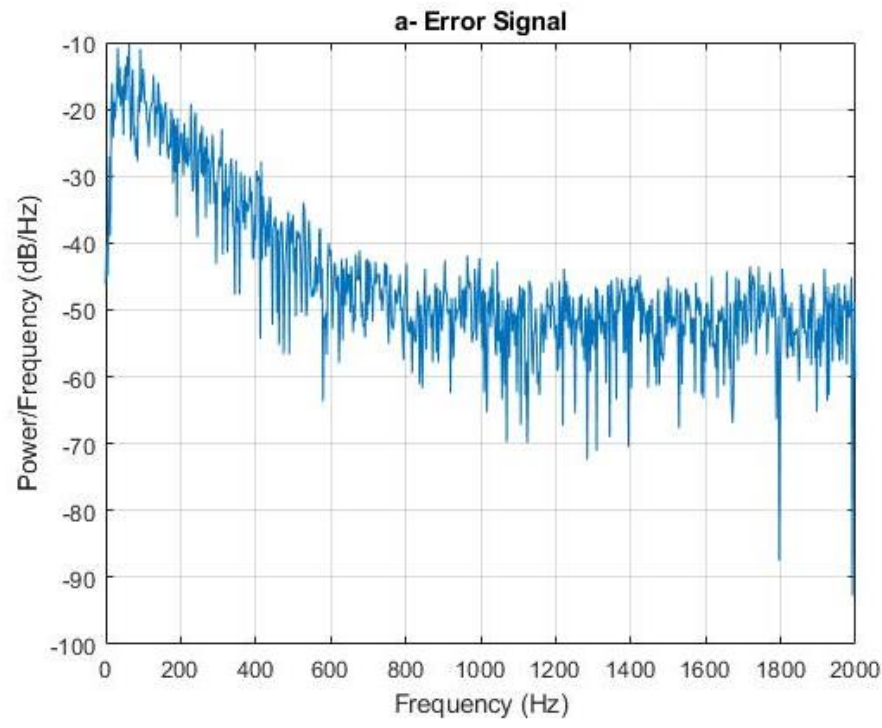

(a)

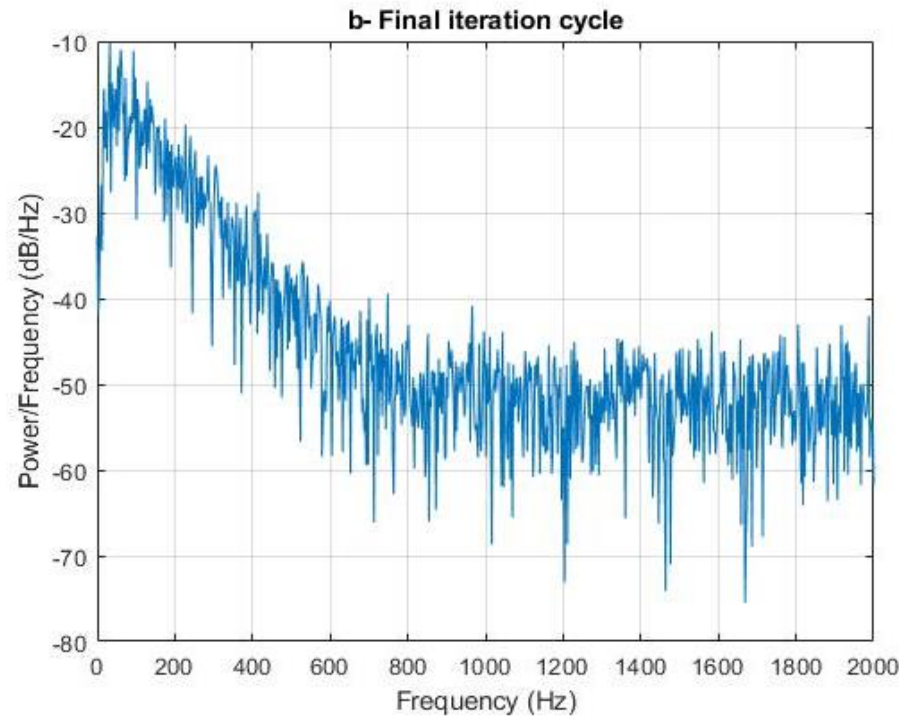

(b)

Figure 5. The power spectrum adopting ESV distribution: (a) error signal, (b) final iteration 


\section{CONCLUSION}

Provide the investigation tests on the real and simulated EMG signals according to the suggested filters of different muscles and windows. By the comparison of the results of the robust proposed method as compared with conventional methods, we can enumerate the following conclusions: our results show that the RV technique gives the optimal approximation of the model order among the other suggested studied techniques, regardless of the type of muscles and investigation time that was not considered by baseline studies. White noise error of the simulated EMG signal has slight progress over the power spectrum estimation. Consequently, the weighted filtering function's significance related to one of the symmetrical distribution is known as the extreme largest distribution. Meanwhile, the assumption of normality to the simulated white noise error signal has yielded. Progress at $400 \mathrm{msec}$ window. This improvement that the filtering of asymmetrical type of contamination distribution (extreme value distribution-largest values) and the modification that the assumption of normality for the pre-white noise has been strengthed. The suggested robust method improved the principle of robustness. The obtained results agree with that of conventional results, the window of the studied cases; meanwhile, it gives more accurate results than the traditional method.

\section{REFERENCES}

[1] M. Gonzalez-Izal, E. L. Cadore, and M. Izquierdo, "Muscle conduction velocity, surface electromyography variables, and echo intensity during concentric and Eccentric fatigue," in Muscle and Nerve, vol. 49, no. 3, pp. 389-397, Jul. 2013, doi: 10.1002/mus.23926.

[2] S. Borbély, A. Toth, and L. Nagy, "Spatial and temporal interference during the ionization of H by few-cycle XUV laser pulses," in Physical Review A, vol. 87, no. 1, Jan. 2013, doi: 10.1103/PhysRevA.87.013405.

[3] P. Huang et al., "Identification of upper-limb movements based on muscle shape change signals for human-robot interaction," in Computational and Mathematical Methods in Medicine, vol. 2020, Apr. 2020, Art. no. 14, doi: 10.1155/2020/5694265.

[4] A. Ibrahim, "A multi-function MyoController for upper limb prostheses," in 18th International Conference on Engineering Systems in Medicine and Biology, Berlin, 2016.

[5] A. Adam and C. J. Luca, "Firing rates of motor units in human vastus lateralis muscle during fatiguing isometric contractions," Journal of Applied Physiology, vol. 99, no. 1, Jul. 2005, doi: 10.1152/japplphysiol.01344.2004.

[6] A. F. Siegel et al., "Practical Business Statistics," in Academic Press, 2017.

[7] N. V. Torres and S. Guido, "The (mathematical) modeling process in biosciences," in Frontiers in Genetic, vol. 6, no. 354, Dec. 2015, doi: 10.3389/fgene.2015.00354

[8] D.-Klug, J. Silny and G. Rau, "Improvement of spatial resolution in surface-EMG: a theoretical and experimental comparison of different spatial filters," in IEEE Transactions on Biomedical Engineering, vol. 44, no. 7, pp. 567-574, Jul. 1997, doi: $10.1109 / 10.594897$

[9] K. A. AL-Naqeeb and A. A. Ibrahim, "Power spectra robust estimation of ECG signals using autoregressive simulation," in 2nd International Conference on Biomedical Engineering and July systems, Barcelona, 2015.

[10] Y. Nardi and A. Rinaldo "Autoregressive process modeling via the lasso procedure," in Journal of Multivariate Analysis, vol. 102, no. 3, pp. 528-549, 2011, doi: 10.1016/j.jmva.2010.10.012.

[11] L. F. Sommer, C. Barreira, C. Noriega, F. Camargo-Junior, R. T. Moura and A. F.-Cordero, "Elbow joint angle estimation with surface electromyography using autoregressive models," 2018 40th Annual International Conference of the IEEE Engineering in Medicine and Biology Society (EMBC), 2018, pp. 1472-1475, doi: 10.1109/EMBC.2018.8512512.

[12] A. Furui, H. Hayashi, Y. Kurita and T. Tsuji, "Variance distribution analysis of surface EMG signals based on marginal maximum likelihood estimation," 2017 39th Annual International Conference of the IEEE Engineering in Medicine and Biology Society (EMBC), 2017, pp. 2514-2517, doi: 10.1109/EMBC.2017.8037368.

[13] H.-Wright and D. W. Stashuk, "Improved MUP template estimation using local time warping and kernel weighted averaging," 2018 40th Annual International Conference of the IEEE Engineering in Medicine and Biology Society (EMBC), 2018, pp. 26472650, doi: 10.1109/EMBC.2018.8512886.

[14] L. F. Sommer and A. Forner-Cordero, "Elbow joint angle estimation from surface electromyography using Hammerstein-wiener models," 2018 7th IEEE International Conference on Biomedical Robotics and Biomechatronics (Biorob), 2018, pp. 1044-1049, doi: 10.1109/BIOROB.2018.8487940.

[15] J. C. Kline and C. J. De Luca, "Error reduction in EMG signal decomposition," in Journal of Neurophysiology, vol. 112, 2013, doi: $10.1152 /$ jn. 00724.2013

[16] D. J. Thomson, "WT4 millimeter waveguide system: spectrum estimation techniques for characterization and development of WT4 waveguide-II," The Bell System Technical Journal, vol. 56, no. 10, pp. 1983-2005, 1977, doi: 10.1002/j.15387305.1977.tb00164.x.

[17] E. Stålberg et al., "Standards for quantification of EMG and neurography," in Clinical Neurophysiology, vol. 130, no. 9, 2019, doi: 10.1016/j.clinph.2019.05.008.

[18] T. Chen, E. Martin, and G. Montague, "Robust probabilistic PCA with missing data and contribution analysis for outlier detection," in Computational Statistics and Data Analysis, vol. 53, pp. 3706-3716, Aug. 2009, doi: 10,1016/j.csda.2009.03.014.

[19] A. AL-Naqeeb, "Robust estimation of power spectra with application," Ph.D. Dissertation, University of Baghdad, Iraq, 1997.

[20] I. Meigal et al., "Novel parameters of surface EMG in patients with Parkinson's disease and healthy young and old controls," in Journal of Electromyography and Kinesiology, vol. 19, no. 3, 2009, doi: 10.1016/j.jelekin.2008.02.008.

[21] P. Konrad, "The ABC of EMG: a practical introduction to kinesiological electromyography," Noraxon Inc., USA, Apr. 2005.

[22] M. M. Rahman, "Analysis of 2D spatial filtering of simulated muscle action potential using grid arrays," M.S. thesis, Electrical Engineering, University of Tennessee, United States, 2005.

[23] H. Reucher, G. Rau and J. Silny, "Spatial filtering of noninvasive multielectrode EMG: Part I-introduction to measuring technique and applications," in IEEE Transactions on Biomedical Engineering, vol. BME-34, no. 2, pp. 98-105, Feb. 1987, doi: 10.1109/TBME.1987.326034. 
[24] E. J. Hannan and B. G. Quinn, "The determination of the order of an autoregression," Journal of the Royal Statistical Society, vol. 41, pp. 190-195, 1979.

[25] A. Boardman, F. S. Schlindwein, A. P. Rocha, and A. Leite, "A study on the optimum order of autoregressive models for heart rart variability," Institute of Physics Publishing, Physiol Measurement, vol. 23, no. 2, 2002.

[26] A. Atyabi, F. Shic, and A. Naples, "Mixture of autoregressive modeling orders and its implication on single-trial EEG classification," Expert Systems with Applications, vol. 65, pp. 164-180, 2016, doi: 10.1016/j.eswa.2016.08.044.

\section{BIOGRAPHIES OF AUTHORS}

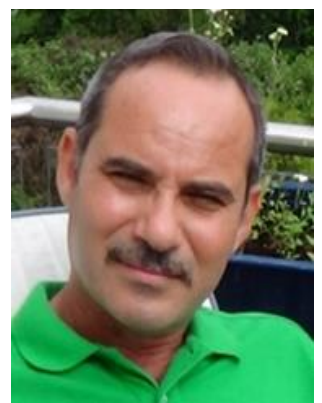

Ayad Asaad Ibrahim (D) SI SC P has received his B.Sc. And M. Sc., degrees in 1982 and 1984 respectively from the University of Technology, Baghdad. He is currently a Lecturer in the Department of Electrical Engineering, University of Technology, Baghdad. His research interest is Biomedical Engineering and EMG signal processing. He has many publications in national and international journals. Also, participate in many international conferences in these mentioned fields. He can be contacted at email: 30055@uotechnology.edu.iq.

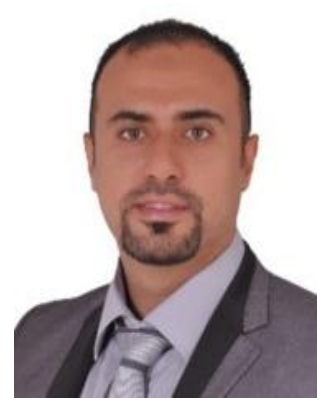

Mohammed Ehsan Safi (iD) SC P Pas received his B.Sc. and M. Sc. degrees in 2008 and 2013, respectively, from the University of Technology, Baghdad. He is currently a Lecturer in the Department of Electrical Engineering, University of Technology, Baghdad. His research interest is Pattern Recognition, Image process, Biomedical: EMG signal processing, and smart system design. He has many publications in National and International Journals. Also, participate in many International Conferences in these mentioned fields. He can be contacted at email: 30165@uotechnology.edu.iq.

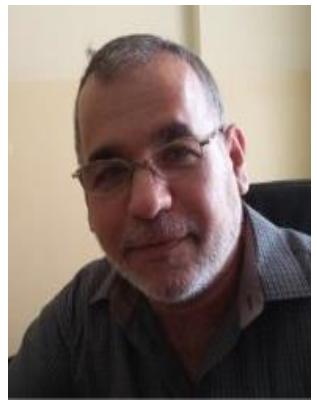

Eyad Ibrahim Abbas (D) 8 sc P has received his B.Sc., M. Sc., and Ph.D. degrees in 1985, 1998, and 2004 respectively from the University of Technology, Baghdad. He has been Assistant Professor of Electronic Engineering in the Department of Electrical Engineering. He has more than 30 publications in national and international journals. Also, participate in more than 15 international conferences in the field of electronics and communication systems. He can be contacted at email: 30013@uotechnology.edu.iq. 